\title{
Treatment for Head and Neck Cancer
}

\author{
FUMIHIKO MATSUMOTO* \\ *Department of Otorhinolaryngology, Juntendo University Faculty of Medicine, Tokyo, Japan
}

\begin{abstract}
The head and neck have important functions, including speech, articulation, swallowing, mastication, and cosmesis. The treatment of head and neck cancer (HNC) results in the impairment of these functions. Therefore, it is necessary to consider the balance between curability and post-treatment quality of life in the treatment of HNC. Current knowledge about the treatment of HNC is necessary for head and neck surgeons. This study focuses on new topics in HNC treatment, including radiotherapy and concomitant intra-arterial cisplatin, perforator skin flap, human papilloma virus, transoral robotic surgery, narrow band imaging, transoral surgery, voice prosthesis, active surveillance for micropapillary carcinoma, particle beam radiotherapy, and drug development: immune checkpoint inhibitors, molecular target drugs, and photoimmunotherapy.
\end{abstract}

Key words: head and neck cancer (HNC), trans oral surgery, immune check point inhibitor, human papilloma virus (HPV)

\section{Introduction}

Head and neck cancer (HNC) is a malignant tumor that develops from the head to the clavicular region, except for the brain and eyeball, and includes cancers of the tongue, pharynx, larynx, parotid, and thyroid regions. The frequency of HNC is approximately $3 \%$ of all cancers. According to the 2016 Japanese Head and Neck Cancer Society National Registry of Head and Neck Malignancies, the most common type of cancer is oral cavity cancer, followed by laryngeal, hypopharyngeal, and oropharyngeal cancers. HNC is frequent worldwide. In India, oral cancer is particularly common and has been linked to chewing tobacco. In the southern part of China, there are many patients with nasopharyngeal cancer due to the Ebstein-Barr virus. These factors make it the sixth most common cancer worldwide. Other regional characteristics include oropharyngeal carcinoma derived from human papilloma virus in the United States, laryngeal carcinoma in Europe where there is a relatively large smoking population, and hypopharyngeal carcinoma in Japan and Korea where the activity of aldehyde dehydrogenase is low. The head and neck region is closely linked to human life and is responsible for functions such as occlusion, mastication, swallowing, articulation, speech, and esthetics. Impairing these functions can greatly reduce the quality of life (QOL). Therefore, in the management of HNC, balance between post-treatment, QOL, and radicalism is considered. The curative treatments for HNC include surgery and radiotherapy. Chemotherapy is only an adjunct to these therapies, and it is difficult to achieve complete healing with chemotherapy alone. In the case of surgery, although a larger resection is more curative, postoperative functional impairment is greater. Therefore, it is also necessary to consider the balance between curability and post-treatment QOL in the treatment of HNC. Hence, current knowledge about HNC treatment is necessary for

\footnotetext{
Fumihiko Matsumoto

Department of Otorhinolaryngology, Juntendo University Faculty of Medicine

2-1-1 Hongo, Bunkyo-ku, Tokyo 113-8421, Japan

TEL: +81-3-5802-1229 FAX: +81-3-5840-7103 E-mail: fmatsu@juntendo.ac.jp

〔Received Feb. 18, 2021〕〔Accepted Mar. 3, 2021〕
}

Copyright (C) 2021 The Juntendo Medical Society. This is an open access article distributed under the terms of Creative Commons Attribution License (CC BY), which permits unrestricted use, distribution, and reproduction in any medium, provided the original source is properly credited. doi: 10.14789/jmj.2021.67.JMJ21-LN01 
head and neck surgeons. In this study, the current findings necessary to make the right decisions in the diagnosis and treatment of HNCs are discussed.

\section{New topics in the treatment for head and neck cancer}

\section{1) Radiotherapy and concomitant intra-arterial cisplatin (RADPLAT)}

In advanced maxillary sinus cancer, total maxillectomy making a skin incision on the face and reconstruction surgery with free flap after resection are required. Total maxillectomy is associated with several postoperative morbidities, including loss of teeth, malocclusion, numbness, and scar formation on the face. However, in recent years, a new treatment method, RADPLAT, has been developed to replace total maxillectomy to maintain QOL after treatment. In RADPLAT, the catheter is approached from the femoral artery, passed through the carotid artery, and finally advanced to the feeding artery of the tumor. In the case of maxillary sinus cancer, the maxillary artery is the main feeding artery. Once the tip of the catheter is placed in the feeding artery, the anticancer drug cisplatin is administered. At the same time, sodium thiosulfate is administered through a catheter inserted into the subclavian vein to neutralize cisplatin. This method makes it possible to administer a large amount of cisplatin to the tumor while reducing the systemic side effects of the neutralizing agent ${ }^{1)}$ (Figure-1).

\section{2) Perforator skin flap}

In the surgical treatment for advanced $\mathrm{HNC}$, extended resection is required. After a wide resection, the surgical defect is reconstructed with a free flap; otherwise, postoperative functional impairment will be significant. In the past, the rectus abdominis and forearm flap were commonly used for reconstruction. However, a perforator skin flap has recently been developed. The anterolateral thigh flap is one of the perforator flaps fed by the descending branch of the lateral circumflex femoral artery. The benefit of this flap is that is it prevents muscle destruction, unlike the traditional skin flaps, and the harvest site can be sutured primarily.

\section{3) Human papilloma virus (HPV)}

The main risk factors for oropharyngeal cancer (OPC) are smoking and alcohol consumption. The disease is more common in elderly men with a history of smoking and alcohol consumption. HPV is involved in the carcinogenesis of OPC. The number of patients who develop OPC due to HPV is increasing, and it occurs frequently in young women without a history of drinking or smoking. HPV is a sexually transmitted infection, and many people are infected at least once in life. It is usually eradicated, but some people develop cancer from
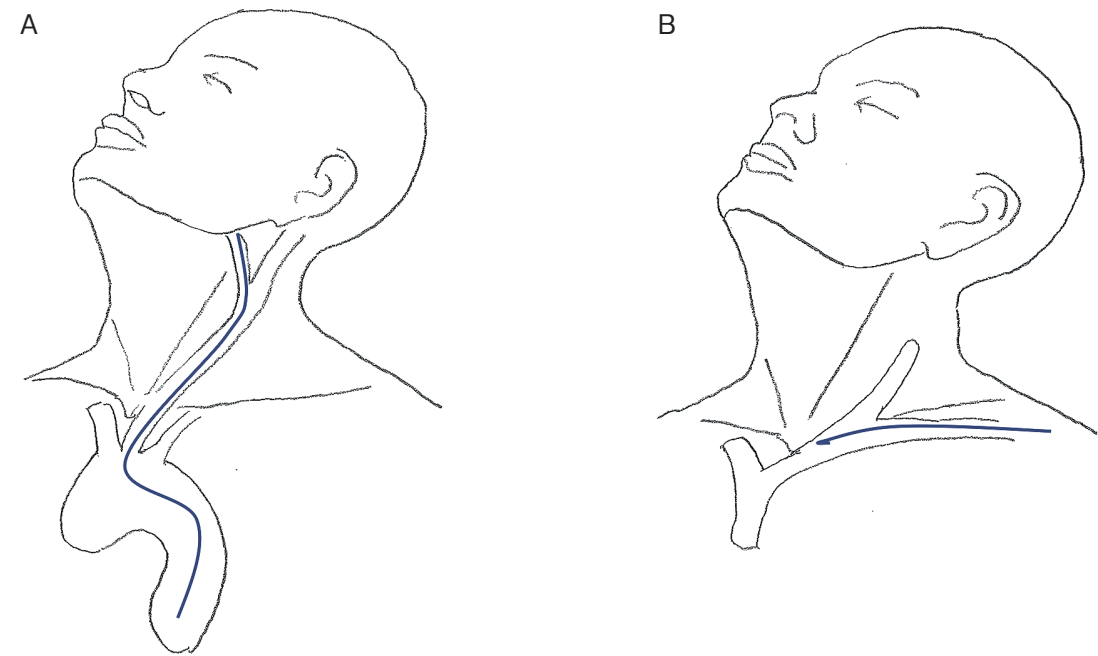

Figure-1 The mechanism of RADPLAT

The catheter is advanced to the feeding artery of the tumor. A high volume of cisplatin is administered from the catheter (A). Sodium thiosulfate is administered through a catheter inserted into the subclavian vein to neutralize cisplatin (B). 
persistent infection, similar to cervical cancer. The mechanism of persistent infection in the oropharynx remains unknown. HPV-related oropharyngeal carcinoma develops cervical lymph node metastasis in the early phase. Because lymph node metastases often have a cystic appearance, they are diagnosed as cystic diseases such as lateral cervical cysts. If a cystic mass is found in the neck, the oropharynx should be checked. p16 immunochemical staining is a surrogate marker for HPV association. HPVrelated OPC is characterized by high sensitivity to radiation and chemotherapy. It has been reported that the prognosis is better than that of non-HPVrelated OPC. Therefore, in the latest edition of the TNM classification, HPV-related OPC is distinguished from non-related OPC and the classification is defined ${ }^{2)}$.

\section{4) Transoral robotic surgery}

Robotic surgery is becoming more popular in the treatment of HNC. The main advantage of robotic surgery is that it is possible to resect pharyngeal cancer using only the transoral approach without skin incision. It is particularly useful for OPC. The diameter of the robotic arm is as thin as $5 \mathrm{~mm}$, and the tip has a $360^{\circ}$ range of motion; therefore, it can be operated from various angles even in the narrow area of the mouth ${ }^{3)}$.

\section{5) Narrow band imaging (NBI)}

HNC has a high frequency of multiple cancers, especially HPC, and approximately $20 \%$ of HPCs are associated with esophageal cancer. Recently, the early detection of HPC has increased due to upper gastrointestinal endoscopy for the follow-up of esophageal and gastric cancer. This is largely due to the introduction of the NBI technology. NBI is a technology that narrows the range of the wavelengths of irradiated light to emphasize minute changes in the irradiated surface. Since cancer cells grow rapidly by supplying nutrients from blood vessels, small blood vessels tend to gather in the cancerous area. NBI can be an effective diagnostic method in the detection of microscopic mucosal patterns on the surface of the mucosa. By observing these images under magnification, cells with abnormal morphology, such as cancer cells, can be detected at an early stage ${ }^{4)}$.

\section{6) Transoral surgery}

In early cases that are diagnosed by NBI, less invasive resection can be performed. Robotic surgery is not yet covered by insurance in Japan. For instance, with the development of surgical devices and techniques, transoral resection, endoscopic laryngeal-pharyngeal surgery, and transoral video-assisted surgery have become standard procedures in recent years. The indications for this procedure have expanded to include not only mucosal lesions but also those with a certain tumor volume. With these methods, oral minimally invasive resection is possible, and treatment can be performed in a short period of time, similar to robotic surgery. Traditionally, radiotherapy has been delivered for early pharyngeal carcinoma. It causes adverse reactions such as loss of taste and mucositis. However, transoral resection achieves complete resection while being minimally invasive $^{5)}$.

\section{7) Voice prosthesis}

In HPC or laryngeal cancer, extended resection and total laryngectomy are required for advanced cases. Total laryngectomy results in the loss of voice. In the past, the substitute voice after total laryngectomy was artificial larynx or esophageal vocalization, but now voice prosthesis has become popular. Voice prosthesis is a small silicone device.

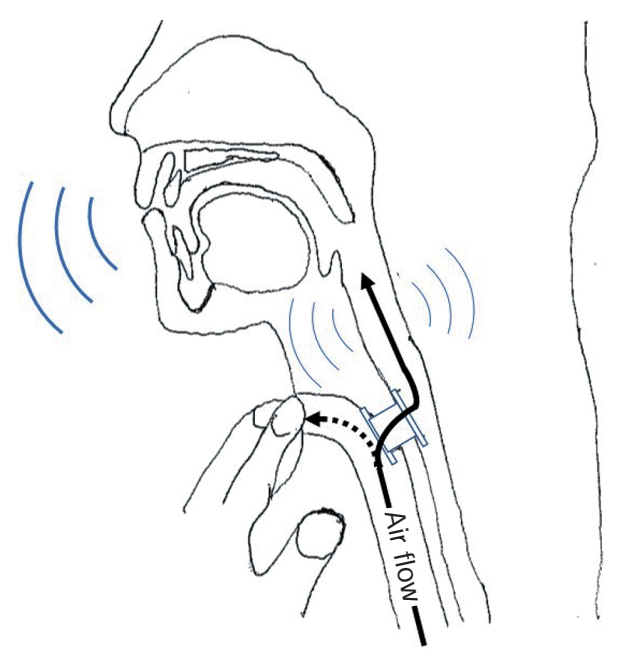

Figure-2 The mechanism of voice prosthesis By blocking the permanent tracheal hole with a finger when exhaling, exhaled air flows from the trachea to the pharynx. Speech is produced by shaking the pharynx as exhaled air flows through the pharynx and oral cavity. 
It is inserted from the posterior wall of the permanent tracheal fistula to the esophagus. It has a one-way valve, and air flows from the trachea in the direction of the esophagus, but food and drinks do not flow in the opposite direction. By blocking the permanent tracheal hole with a finger when exhaling, exhaled air flows from the trachea to the pharynx. Speech is produced by shaking the pharynx as exhaled air flows through the pharynx and oral cavity. This method enables voice communication and maintains the postoperative QOL $(\text { Figure }-2)^{6)}$.

\section{8) Active surveillance for micropapillary carci- noma}

Thyroid cancer is an indolent cancer with a 10year survival rate of papillary thyroid carcinoma which is more than 90\%. When autopsies are performed on people who have died from other causes, thyroid cancer is found in approximately 1 out of every 10 cases. In contrast, only about 3 out of 100,000 people undergo surgery for papillary thyroid cancer per year. These facts show that many people who suffer from papillary carcinoma live their lives without experiencing its manifestation. Papillary carcinomas with a tumor size of $<10$ $\mathrm{mm}$ and without lymph node metastasis or symptoms are defined as micropapillary carcinomas (MPCs). MPCs grow in less than $10 \%$ of cases even after a long follow-up. Even if surgery is performed when the tumor has grown to $>10 \mathrm{~mm}$, the prognosis does not deteriorate, and no distant metastasis occurs. For this reason, the current policy in Japan for MPC is active surveillance (AS), which is a follow-up observation for early detection of tumor growth. In cases where the patient desires surgery, surgical resection is performed. AS should be undertaken only after obtaining an explanation and consent from the patient ${ }^{7)}$.

\section{9) Particle beam radiotherapy}

Particle beam therapy is a radiotherapy particle beam and includes protons or carbon ions. This therapy has the advantage of being more targeted to cancer lesions than conventional treatment with $\mathrm{X}$-rays. Currently, in addition to children and sarcomas, non-squamous cell carcinoma of the head and neck and nasal sinus cancer (any pathological type) are covered by insurance. In conventional radiotherapy, the radiation dose is greatest near the surface of the body, and after that, the amount of absorbed radiation gradually decreases as it enters the body. Unidirectional irradiation is used to sufficiently damage deep cancerous lesions, causing more damage to normal cells that are shallower than cancerous lesions. In contrast, protons and carbon ions do not emit energy near the surface of the body when they enter the body, emitting energy just before they stop to deliver a large dose to the tissue (Bragg peak). By adjusting the depth and width of this peak according to the depth and size of the lesion, the radiation dose focuses only on the lesion, thereby reducing the dose to normal tissues $^{8)}$.

\section{0) Development drug treatment: Immune check} point inhibitor, molecular target drug

The following is a list of relatively new drugs that are currently approved for head and neck cancers. Multi-kinase inhibitors, lenvatinib and sorafenib, are approved for thyroid cancer and used for distant metastasis or recurrence of thyroid cancer (Table-1). Lenvatinib has proven to be effective in anaplastic carcinoma and has been administered to patients with unresectable anaplastic carcinoma. The monoclonal antibody cetuximab is an antiEGFR antibody that works by binding to the EGRFR on the surface of cancer cells. It has been shown to be effective in combination with radiotherapy for HNC or in combination with chemotherapy for distant metastasis or recurrence. In recent years, the field of immune checkpoint inhibitors has seen remarkable development. Cancer cells bind to PD- 1 antibodies on the surface of T cells via PD-L1 receptors, thereby inactivating $\mathrm{T}$ cells and avoiding elimination by the immune system. The mechanism

Table-1 New drug for head and neck cancer

\begin{tabular}{lcc}
\hline Immune check point inhibitor & Multi-kinase inhibitor & Monoclonal antibody \\
\hline Nivolumab & Lenvatinib & Cetuximab \\
Pembrolizumab & Sorafenib & \\
\hline
\end{tabular}


of immune checkpoint inhibitors is to avoid T-cell inactivation by binding to the $\mathrm{PD}-1$ receptor on the T-cell surface. Nivolumab and pembrolizumab are immune check inhibitors that are approved for the metastatic or recurrence of $\mathrm{HNC}$. The response rate with these treatments is low and is reported to be $13.3 \%$. In patients who responded to nivolumab, the effect lasts for a long time in many cases. Even if the drug is ineffective, there are many cases in which chemotherapy after an immune checkpoint inhibitor is successful. The mechanism is not yet clear, but it is speculated that changes in the immune environment may increase the effectiveness of these drugs. High medical costs are a problem with immune checkpoint inhibitors, with a typical dose costing 300,000 yen per month. Another problem is that a wide variety of immune-related adverse events, such as fulminant hepatitis or severe diabetes, may suddenly occur ${ }^{9)}$ 10).

\section{1) Photoimmunotherapy}

A recent treatment is photoimmunotherapy. Antibodies that recognize cancer cells are immersed in a chemical that generates heat when exposed to near-infrared light and injected directly into the tumor. The tumor is then irradiated with near-infrared radiation. Near-infrared radiation reacts with the substance mentioned above and generates heat, which destroys the cancer cells. Currently, we are using antibodies that target EGFR, which is expressed in more than $90 \%$ of head and neck cancers. Head and neck cancers that are closer to the body surface are good indications because of the injection of antibodies into the tumor and the irradiation of near-infrared radiation. Therefore, this treatment is more developed for head and neck cancer. Since this treatment method has been approved by the Japanese government, it is expected to gradually become popular in Japan.

\section{Acknowledgments}

None.

\section{Conflict of interest}

The Author declares that there are no conflicts of interest.

\section{References}

1) Robbins KT, Kumar P, Regine WF, et al: Efficacy of targeted supradose cisplatin and concomitant radiation therapy for advanced head and neck cancer: the Memphis experience. Int J Radiat Oncol Biol Phys, 1997; 38: 263-271.

2) Ang KK, Harris J, Wheeler R, et al: Human papillomavirus and survival of patients with oropharyngeal cancer. N Engl J Med, 2010; 363: 24-35.

3) Hassaan A, Trinidade A, Kotecha B, Tolley N: TORS for OSA: a practice, pitfalls and literature review. Int J Health Care Qual Assur, 2019; 32: 488-498.

4) Ni XG, Wang GQ: The Role of Narrow Band Imaging in Head and Neck Cancers. Curr Oncol Rep, 2016; 18: 10.

5) Tomifuji M, Araki K, Yamashita T, Shiotani A: Transoral videolaryngoscopic surgery for oropharyngeal, hypopharyngeal, and supraglottic cancer. Eur Arch Otorhinolaryngol, 2014; 271: 589-597.

6) Fukushima H, Kanazawa T, Kawabata K, et al: Indwelling voice prosthesis insertion after total pharyngolaryngectomy with free jejunal reconstruction. Laryngoscope Investig Otolaryngol, 2017; 2: 30-35.

7) Ito $\mathrm{Y}$, Miyauchi A, Oda H: Low-risk papillary microcarcinoma of the thyroid: A review of active surveillance trials. Eur J Surg Oncol, 2018; 44: 307-315.

8) Thariat J, Bolle S, Demizu Y, et al: New techniques in radiation therapy for head and neck cancer: IMRT, CyberKnife, protons, and carbon ions. Improved effectiveness and safety? Impact on survival? Anticancer Drugs, 2011; 22: 596-606.

9) Burtness B, Harrington KJ, Greil R, et al: Pembrolizumab alone or with chemotherapy versus cetuximab with chemotherapy for recurrent or metastatic squamous cell carcinoma of the head and neck (KEYNOTE-048): a randomised, open-label, phase 3 study. Lancet, 2019; 394: $1915-1928$.

10) Ferris RL, Blumenschein G Jr, Fayette J, et al: Nivolumab for Recurrent Squamous-Cell Carcinoma of the Head and Neck. N Engl J Med, 2016; 375: 1856-1867. 